\title{
Combination of Ascorbic Acid and Methylprednisolone Pulse Therapy in the Treatment of Idiopathic Thrombocytopenic Purpura
}

\author{
Jiro Masugi, Masahide Iwai, Sachiko Kimura, Fukashi Ochi, Kotaro Suzuki, \\ Osamu Nakano, Taizo SaKamoto, Hideyuki Fukunaga, Masahiko Amano and Tsuneo Fukuda
}

\begin{abstract}
Ascorbic acid, reported in 1988 to be effective for idiopathic thrombocytopenic purpura (ITP), is an attractive drug because of its lack of toxicity. Further studies are necessary in order to improve its effectiveness without increasing secondary effects. We present a chronic ITP patient treated with a combination of ascorbic acid and methylprednisolone pulse (MP) therapy who was previously treated with MP therapy alone. The effect of this combination therapy seems to be better than MP therapy alone. This therapy is worth further examination as another therapeutic choice due to its fewer secondary effects than the usual regimen of corticosteroids, splenectomy, and other immunosuppressive drugs.
\end{abstract}

(Internal Medicine 33: 165-166, 1994)

Key words: ascorbate, corticosteroid, immuno-suppressive drug, interferon, splenectomy

\section{Introduction}

The most common drugs for idiopathic thrombocytopenic purpura (ITP) are corticosteroids or immuno-suppressive drugs, but with long-term administration, the adverse effects often become a problem. Ascorbic acid is an attractive drug because of its lack of toxicity (1), but its effectiveness is not that high. An effective means of administration of ascorbic acid necessitates further study.

Recently we observed the effect of the combination of ascorbic acid and methylprednisolone pulse (MP) therapy for a chronic ITP patient. Ascorbic acid seemed to enhance the effect of methylprednisolone.

\section{Patient}

A 69-year-old woman was admitted to our hospital because of purpura. Physical examination was negative except for the purpura on both arms. Peripheral blood showed thrombocytopenia with a platelet count of $1.8 \times 10^{4} / \mathrm{mm}^{3}$ with normal white blood cell and red blood cell counts. Blood chemistry was unremarkable. Platelet-associated $\operatorname{IgG}(\mathrm{PA} \operatorname{IgG})$ was as high as $366.2 \mathrm{ng} / 10^{7}$ cells. Bone marrow examination revealed normal marrow, with a normal count of megakaryocytes and no atypical cells. A diagnosis of ITP was made. At first, she was treated with MP therapy (a single intravenous injection of methylprednisolone, $1 \mathrm{~g} /$ day for three consecutive days) twice with the expectation of a quick response, but the effect was transient and lasted only approximately three weeks. The increase in the platelet count with the second MP therapy was lower than that of the first. Then $2 \mathrm{~g}$ of ascorbic acid was administered orally in three doses per day and the third MP therapy was administered. The response of the third MP therapy was better than that of the first and the second, and the platelet count of $3 \times 10^{4}-4 \times 10^{4} / \mathrm{mm}^{3}$ lasted for about one month. Then MP therapy was performed again; this time it had a higher and prolonged effect (Fig. 1).

\section{Discussion}

The typical therapy for ITP includes oral corticosteroids and splenectomy. Other modalities are immuno-suppressive drugs, vinca alkaloids, danazol, or alpha interferon. However, even though approximately $60 \%$ of patients respond to oral corticosteroids, only $20-30 \%$ achieve complete remission. Immuno-suppressive drugs have a lower remission rate, and these therapies have severe side effects $(2,3)$. Other therapeutic choices are intravenous gammaglobulins (4) or methylprednisolone pulse therapy (5), but the effects of these are usually transient and the former is quite expensive.

From the Department of Medicine, Nishiwaki Municipal Hospital, Nishiwaki

Received for publication August 26, 1993; Accepted for publication January 6, 1994

Reprint requests should be addressed to Dr. Jiro Masugi, the Department of Medicine, Nishiwaki Municipal Hospital, 652-1 Shimotoda, Nishiwaki, Hyogo 677 


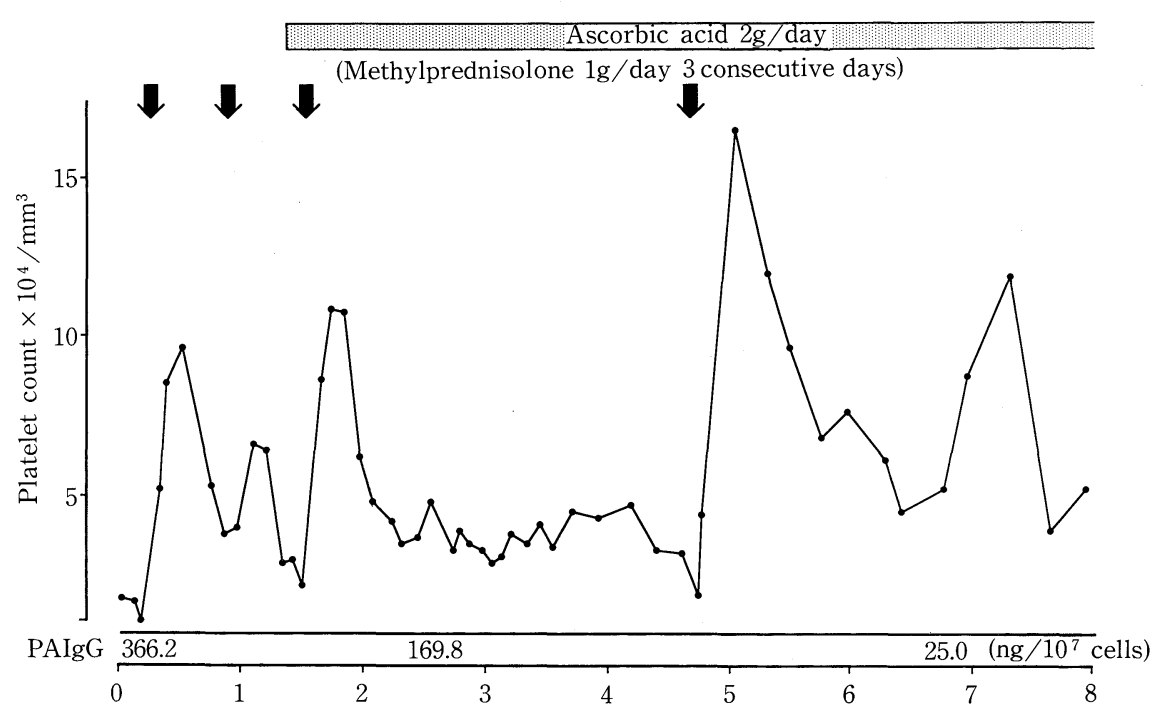

Fig. 1. Clinical course (months) of the patient. Methylprednisolone pulse therapy was more effective after the administration of ascorbic acid than before administration of ascorbic acid.

In 1988, Brox et al (1) reported that ascorbic acid is effective for refractory ITP. They reported that 9 out of their 11 patients $(82 \%)$ showed a satisfactory increase in platelet counts by taking $2 \mathrm{~g}$ of ascorbic acid as a single daily dose. They concluded that ascorbic acid might be an attractive therapeutic option in the treatment of ITP. But there are no reports to date which confirm this high response. Vianelli et al (6) analyzed 55 case reports in the literature and some of their own cases, and showed that the incidence of complete response and partial response is 5\% and 15\%, respectively. The ITP Research Group of Japan sponsored by the Welfare Ministry reported that only $11.5 \%$ of 78 refractory ITP patients showed minimal to partial response to ascorbic acid therapy (7).

The reason the results are so different is unclear. It may be because the patients of Brox et al (1) were generally younger (6), or because most of their patients took $2 \mathrm{~g}$ of ascorbic acid or more at the same time before meals (8).

The effect of ascorbic acid on ITP is not known. Brox revealed that platelet survival is prolonged (1), but the mechanism of its action is unknown. It may be partly explained by the fact that ascorbic acid enhances the production of interferon (9), which is effective for ITP (10). In the present case, when the platelet count was increased, PA IgG was decreased. Ascorbic acid may activate some other cytokines which suppress PA IgG.

Ascorbic acid is a hopeful drug because of its non-toxicity. Further, it is inexpensive. But the disadvantages are its low effectiveness and its tardiness in acting. In the case of Brox's report, 10 weeks on the average were needed for the response. Further studies are needed to determine a means to improve the ascorbate therapy.

In order to compensate for these drawbacks, we adopted MP therapy. The features of MP therapy are quick response and few secondary effects, although the effect is usually transient. In the present case, the effect of MP therapy was greater after the administration of ascorbic acid.

We believe that ascorbic acid and MP therapy are a good combination as each compensates for the other's disadvantages. This combination therapy should be examined further as another therapeutic regimen for ITP because of its potentially fewer secondary effects than the usual regimen of oral corticosteroids, splenectomy, and other immuno-suppressive drugs.

\section{References}

1) Brox AG, Howson-Jan K, Fauser AA. Treatment of idiopathic thrombocytopenic purpura with ascorbate. Br J Haematol 70: 341, 1988.

2) Berchtold P, McMillian R. Therapy of chronic idiopathic thrombocytopenic purpura in adults. Blood 74: 2309, 1989.

3) McMillian R. Chronic idiopathic thrombocytopenic purpura. N Engl J Med 304: 1135, 1981.

4) Imbach P, Barandun S, d'Apuzzo V, et al. High-dose intravenous gammaglobulin for idiopathic thrombocytopenic purpura in childhood. Lancet 1: 1228, 1981

5) Oto A, Sözen T, Özişik Y, et al. Pulse methylprednisolone therapy in idiopathic thrombocytopenic purpura. Acta Haematol. 70: 345, 1983.

6) Vianelli N, Gugliotta L, Gianni L, et al. Ascorbic acid for the treatment of chronic refractory idiopathic thrombocytopenic purpura. Haematologica 77: 92, 1992.

7) Kuramoto J, Fujimura K, Takada N. Effects of ascorbate therapy for refractory idiopathic thrombocytopenic purpura: multi-center joint pilot study. A report on research and achievement in the specific disease, "idiopathic dyshematopoiesia", submitted in 1989 by the Survey and Research Group of the Welfare Ministry, March: 124, 1990 (in Japanese).

8) Brox AG. Ascorbate for the treatment of refractory idiopathic thrombocytopenic purpura. Br J Haematol 74: 234, 1990.

9) Dahl H, Denré M. The effect of ascorbic acid on production of human interferon and the antiviral activity in vitro. Acta Path Microbol Scand Sect B 84: 280, 1976.

10) Proctor SJ, Jackson G, Carey P, et al. Improvement of platelet counts in steroid-unresponsive idiopathic immune thrombocytopenic purpura after short-course therapy with recombinant $\alpha 2 b$ interferon. Blood 74: 1894, 1989. 